\title{
An energy conduction model for cell image segmentation
}

\author{
MA JingFeng ${ }^{1}$, BU JiaJun ${ }^{1}$, HOU Kai ${ }^{2}$, BAO ShangLian ${ }^{2,3}$ \& CHEN Chun ${ }^{1 *}$ \\ ${ }^{1}$ College of Computer Science and Technology, Zhejiang University, Hangzhou 310027, China; \\ ${ }^{2}$ Beijing Key Laboratory of Medical Physics and Engineering, Peking University, Beijing 100871, China; \\ ${ }^{3}$ Beijing Healthway Technology Limited Company, Beijing 100084, China
}

Received November 18, 2010; accepted December 15, 2010

\begin{abstract}
Cell image segmentation is an essential step in cytopathological analysis. Although their execution speed is fast, the results of cell image segmentation by conventional pixel-based, edge-based and continuity-based methods are often coarse. Fine structures in a cell image can be obtained with a method that quickly adjusts the threshold levels. However, the processing time of such a method is usually long and the final results may be sensitive to intensity differences and other factors. In this article, a new energy model is proposed that synthesizes a differential equation from the conventional and level set methods, and utilizes the nonuniformity property of cell images (e.g. cytoplasms are more uneven than the background). The feasibility and robustness of the proposed model was demonstrated by processing relatively complicated background images of both simulated and real cell images.
\end{abstract}

cell image segmentation, level set, energy conduction model

Citation: $\quad$ Ma J F, Bu J J, Hou K, et al. An energy conduction model for cell image segmentation. Chinese Sci Bull, 2011, 56: 1048-1054, doi: 10.1007/s11434$011-4389-\mathrm{z}$

Since it was first demonstrated in cervical carcinoma cell images by Babes, the analysis of cellular pathology has become increasingly important for the accurate diagnosis and treatment of cancer. Usually, the first step in a successful cytopathological analysis is to segment the nucleolus and cytoplasm from other background organelles. The segmented results are then used in a quantitative analysis of cellular pathology. The objective of our study was to develop a method for obtaining accurate and more reliable segmentation results so as to ensure that subsequent calculations of other parameters are correct.

Conventional methods used for cell image segmentation include pixel-based, edge-based, and continuity-based methods [1-7]. These methods are relatively simple and have fast execution speeds. However, most conventional methods are unable to handle detailed structures and obtain fine segmentation results when cell images contain relatively complex features. The active contour model [8-18] was developed recently and has several notable advantages. With this

*Corresponding author (email: chenc@cs.zju.edu.cn) model, the segmentation result can be achieved at a sub-pixel level. The algorithm can be easily implemented in an energy minimization framework in which prior knowledge of shape and intensity can be incorporated [19]. More importantly, the segmentation results are closed curves, which is beneficial to further processing such as shape analysis and pattern recognition. Active contour models are often divided into two main subclasses: edge-based models [20-24] and region-based models [9,10,25-27]. Edge-based models use local edge information to attract the active contour toward the object boundaries. In contrast, region-based models aim to identify each region of interest using a certain region descriptor to guide the motion of the active contour. In a region-based model, Li [19,28,29] proposed region-scalable fitting energy model to solve the problem of intensity inhomogeneity and accelerate the execution of the algorithm.

When the active contour methods were applied directly to cell image segmentation, we found it was not always possible to achieve satisfactory results in cases where the intensity difference between object and background was minor. In this paper, we first analyzed cell image characteristics, and then 
proposed an energy conduction model where the energy was minimized when the three parts of cell image were well segmented. Finally, a new variational function using the level set framework was constructed to segment cell images.

\section{Characteristic features of cell images}

A human body contains many types of cells. While the shape of these cells can be very different, the basic structures are mostly similar. In general, cells are formed of nucleoli and cytoplasts. The nucleolus is located in the center of a cell and is surrounded by cytoplasts. The density of the nucleolus is higher than that of cytoplasm. Furthermore, because many different molecules are present in cells, the cell density is spatially uneven.

Digital microscopes are typically used to take cell images from cell smears. A cell image has three components: nucleoli, cytoplasts and background. Additionally, the following features can usually be recognized in a cell image: (1) the nucleolus part $\Omega_{\mathrm{n}}$ is located within the cytoplasm part $\Omega_{\mathrm{c}}$, and the cytoplasm part is located within the background part $\Omega_{\mathrm{b}}$, (2) the intensity of the nucleolus $I_{\mathrm{n}}$ is higher than that of the cytoplasm $I_{\mathrm{c}}$, and the intensity of the cytoplasm is higher than that of the background $I_{\mathrm{b}}$, (3) the intensity gradient of nucleolus $G_{\mathrm{n}}$ and cytoplast $G_{\mathrm{c}}$ is bigger than that of background $G_{\mathrm{b}}$ because of the presence of different molecules within the cell (Figure 1).

$$
\begin{gathered}
\Omega_{\mathrm{n}} \subset \Omega_{\mathrm{c}} \subset \Omega_{\mathrm{b}}, \\
I_{\mathrm{b}}<I_{\mathrm{c}}<I_{\mathrm{n}}, \\
G_{\mathrm{c}}, G_{\mathrm{n}}>G_{\mathrm{b}} .
\end{gathered}
$$

We hypothesize that better segmentation results can be obtained if both intensity and intensity gradient information are used in the processing. By combining the information of the original image and of its gradient, we expect that the difference between the cellular components of interest and background can be amplified to allow more reliable segmentation of the different cell components.

\section{Energy conduction model for cell image segmentation}

\subsection{Region-based active contour models}

Let $\Omega \subset R^{2}$ be the image domain, and $I: \Omega \rightarrow R$ be a given gray level image. In [22], the image segmentation problem was formulated by Mumford and Shah as follows: given an image $I$, find a contour $C$ which segments the image into non-overlapping regions. Mumford and Shah proposed the following energy function:

$$
F^{\mathrm{MS}}(u, C)=\int_{\Omega}(u-I)^{2} \mathrm{~d} x+\mu \int_{\Omega \backslash C}|\nabla u|^{2} \mathrm{~d} x+v|C|,
$$

where $u$ is an approximation of the original image and is assumed to be smooth within each of the connected components in the image domain $\Omega$ and is separated by the contour C. $|\nabla u|$ represents the gradient of $u$, and $|C|$ is the length of the contour $C$, and $\Omega \backslash C$ is the area of $\Omega$ that excludes $C$, and $\mu$ and $v$ are two coefficients of the relative parts. In practice, it is difficult to minimize this function.

Tery and Luminita [9] proposed an active contour approach to the MS (Mumford and Shah) problem for the special case in which the image $u$ in function (4) is a piecewise constant (PC) function. For an image I, Chan and Vese proposed to minimize the following function:

$$
\begin{aligned}
F^{\mathrm{PC}}\left(C, c_{1}, c_{2}\right)= & \lambda_{1} \int_{\text {outside }(C)}\left(I-c_{1}\right)^{2} \mathrm{~d} x \\
& +\lambda_{2} \int_{\text {inside }(C)}\left(I-c_{2}\right)^{2} \mathrm{~d} x+v|C|,
\end{aligned}
$$

where outside $(C)$ and inside $(C)$ represent the regions outside and inside the contour $C$, and $c_{1}$ and $c_{2}$ represent two constants that approximate the image intensity in outside $(C)$ and inside $(C)$, and $\lambda_{1}, \lambda_{2} v$ and $v$ are the relative coefficients. In [10], Luminita and Tary subsequently proposed a piecewise smooth (PS) function as

$$
\begin{aligned}
F^{\mathrm{PS}}\left(C, u^{+}, u^{-}\right)= & \lambda_{1} \int_{\text {outside }(C)}\left(I-u^{-}\right)^{2} \mathrm{~d} x \\
& +\lambda_{2} \int_{\text {inside }(C)}\left(I-u^{+}\right)^{2} \mathrm{~d} x \\
& +\mu_{1} \int_{\text {outside }(C)}\left|\nabla u^{-}\right|^{2} \mathrm{~d} x \\
& +\mu_{2} \int_{\text {inside }(C)}\left|u^{+}\right|^{2} \mathrm{~d} x+v|C|,
\end{aligned}
$$

where $u^{+}$and $u^{-}$are the approximations of the image inside and outside of the contour $C$, respectively. Both $u^{+}$and $u^{-}$ are smooth within each of the connected components in the image domain $\Omega$ that are separated by the contour $C$. The
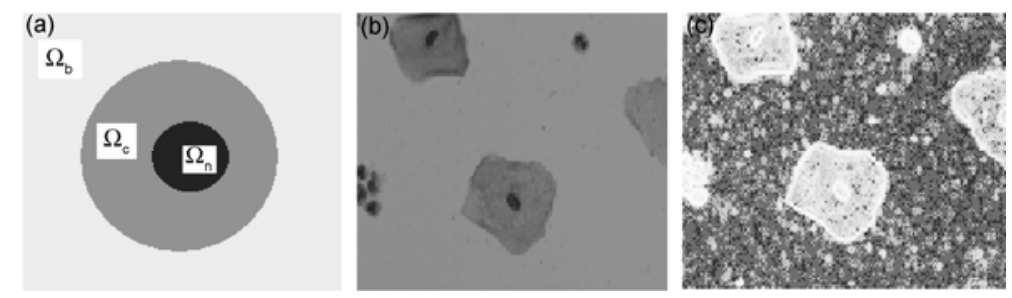

Figure 1 (a) Cell image area; (b) original cell image; (c) gradient of image (b). 
PS model can overcome the limitation of PC models in segmenting images with intensity inhomogeneity. However, the practical application of the PS model is limited because of its high computational load. As a potential solution, Chan and Vese proposed a multiphase level set framework to segment images that contain more than 2 phases [10]. The function that can segment 4 phases is:

$$
\begin{aligned}
F^{\mathrm{PS} 2}\left(c, C_{1}, C_{2}\right)= & \int_{\operatorname{inside}\left(c_{1}\right) \& \operatorname{inside}\left(c_{2}\right)}\left(I-c_{11}\right)^{2} \mathrm{~d} x \\
& +\int_{\operatorname{inside}\left(c_{1}\right) \& \operatorname{outside}\left(c_{2}\right)}\left(I-c_{10}\right)^{2} \mathrm{~d} x \\
& +\int_{\text {outside }\left(c_{1}\right) \& \operatorname{inside}\left(c_{2}\right)}\left(I-c_{01}\right)^{2} \mathrm{~d} x \\
& +\int_{\text {outside }\left(c_{1}\right) \& \operatorname{outside}\left(c_{2}\right)}\left(I-c_{00}\right)^{2} \mathrm{~d} x \\
& +v \int_{\Omega}\left|C_{1}\right|+v \int_{\Omega}\left|C_{2}\right|,
\end{aligned}
$$

in which $C_{1}$ and $C_{2}$ are 2 contours of 2 level set functions, and $c=\left(c_{11}, c_{10}, c_{01}, c_{00}\right)$ is a constant vector with each component representing the approximate constant intensity within each part.

In [19], Li proposed a region-scalable fitting energy function so that the model can accommodate intensity inhomogeneity and accelerate the segmentation process. The function used by $\mathrm{Li}$ is

$$
\begin{aligned}
& F^{\mathrm{RSF}}\left(C, f_{1}, f_{2}\right) \\
& =\sum_{i=1}^{2} \lambda_{i} \int\left(\int_{\Omega_{i}} K_{\sigma}(x-y)\left|I(y)-f_{i}(x)\right|^{2} \mathrm{~d} y\right) \mathrm{d} x+v|C|,
\end{aligned}
$$

in which $\Omega_{1}=\operatorname{outside}(C), \Omega_{2}=\operatorname{inside}(C), f_{1}(x)$ and $f_{2}(x)$ are the approximate image in each part, and $K$ is the kernel function that usually assumes a Gaussian kernel:

$$
K_{\sigma}(u)=\frac{1}{(2 \pi)^{n / 2} \sigma^{n}} \mathrm{e}^{-|u|^{2} / 2 \sigma^{2}}
$$

in which $\sigma>0$.

\subsection{Model for cell image segmentation}

According to the cell image features specified in eqs. (1)-(3), we propose a new energy functional to segment the nucleolus, cytoplast and background. Referring to eqs. (7) and (8), the function we propose is

$$
\begin{aligned}
& F^{\text {Cell }}\left(g_{0}, g_{1}, f_{10}, f_{11}, C_{1}, C_{2}\right) \\
& =\lambda_{1} \iint_{\text {inside }\left(c_{1}\right)}\left(K_{\sigma_{1}}(x-y)\left|I G-g_{1}(x)\right|^{2} \mathrm{~d} y\right) \mathrm{d} x \\
& \quad+\lambda_{2} \iint_{\text {outside }\left(c_{1}\right)}\left(K_{\sigma_{1}}(x-y)\left|I G-g_{0}(x)\right|^{2} \mathrm{~d} y\right) \mathrm{d} x \\
& +\lambda_{3} \iint_{\operatorname{inside}\left(c_{1}\right) \& \operatorname{inside}\left(c_{2}\right)}\left(K_{\sigma_{2}}(x-y)\left|I-f_{11}(x)\right|^{2} \mathrm{~d} y\right) \mathrm{d} x \\
& +\lambda_{4} \iint_{\text {inside }\left(c_{1}\right) \& \operatorname{outside}\left(c_{2}\right)}\left(K_{\sigma_{2}}(x-y)\left|I-f_{10}(x)\right|^{2} \mathrm{~d} y\right) \mathrm{d} x \\
& \quad+v_{1} \int_{\Omega}\left|C_{1}\right|+v_{2} \int_{\Omega}\left|C_{2}\right|
\end{aligned}
$$

where $C_{1}$ is the contour between cytoplasts and background, $C_{2}$ is the contour between cytoplasts and the nucleolus, $I G$ is the combination of the original image and gradient information, $f_{10}$ and $f_{11}$ represent the approximate image of the cytoplast part and the nucleolus part, $g_{0}$ and $g_{1}$ is the approximate image of $I G$, inside $\left(c_{1}\right)$ represents the cell part, outside $\left(c_{1}\right)$ represents the background part, inside $\left(c_{1}\right)$ and $\operatorname{inside}\left(c_{2}\right)$ are the nucleolus part and $\operatorname{inside}\left(c_{1}\right)$ and out$\operatorname{side}\left(c_{2}\right)$ are the cytoplast parts.

The precondition of eq. (10) is that the difference between cytoplasts and background is much larger than that between cytoplasts and the nucleolus in $I G$.

According to the cell feature specified in eq. (1), the 3rd, 4th and 6th terms can be computed after obtaining the results of the 1st, $2 \mathrm{nd}$, and 5 th terms. Therefore, the function in eq. (10) can be divided into 2 separate functions for faster execution:

$$
\begin{aligned}
& F^{\text {Cellbc }}\left(g_{0}, g_{1}, C_{1}\right) \\
& =\lambda_{1} \iint_{\text {inside }\left(c_{1}\right)}\left(K_{\sigma_{1}}(x-y)\left|I G-g_{1}(x)\right|^{2} \mathrm{~d} y\right) \mathrm{d} x \\
& \quad+\lambda_{2} \iint_{\text {outside }\left(c_{1}\right)}\left(K_{\sigma_{1}}(x-y)\left|I G-g_{0}(x)\right|^{2} \mathrm{~d} y\right) \mathrm{d} x \\
& \quad+v_{1} \int_{\Omega}\left|C_{1}\right|
\end{aligned}
$$

and

$$
\begin{aligned}
& F^{\text {Cellcn }}\left(f_{10}, f_{11}, C_{2}\right) \\
& =\lambda_{3} \iint_{\operatorname{inside}\left(c_{1}\right) \& \operatorname{inside}\left(c_{2}\right)}\left(K_{\sigma_{2}}(x-y)\left|I-f_{11}(x)\right|^{2} \mathrm{~d} y\right) \mathrm{d} x \\
& +\lambda_{4} \iint_{\operatorname{inside}\left(c_{1}\right) \& \operatorname{outside}\left(c_{2}\right)}\left(K_{\sigma_{2}}(x-y)\left|I-f_{10}(x)\right|^{2} \mathrm{~d} y\right) \mathrm{d} x \\
& \quad+v_{2} \int_{\Omega}\left|C_{2}\right|
\end{aligned}
$$

\subsection{Evolution of energy conduction function}

In the level set method [8], a contour $C \subset \Omega$ is indicated by the zero level set of a Lipschitz function $\phi: \Omega \rightarrow R$, which is called a level set function. Let $H$ be the Heaviside function, the energy functions (11) and (12) can then be expressed as:

$$
\begin{aligned}
& F^{\text {Cellbc }}\left(g_{0}, g_{1}, \phi_{1}\right) \\
& =\lambda_{1} \int\left(\int\left(K_{\sigma_{1}}(x-y)\left|I G-g_{1}(x)\right|^{2} H\left(\phi_{1}(x)\right) \mathrm{d} y\right) \mathrm{d} x\right. \\
& \quad+\lambda_{2} \int\left(\int\left(K_{\sigma_{1}}(x-y)\left|I G-g_{1}(x)\right|^{2}\left(1-H\left(\phi_{1}(x)\right)\right) \mathrm{d} y\right) \mathrm{d} x\right. \\
& \quad+v_{1} \int \mid \nabla H\left(\phi_{1}(x)\right) \mathrm{d} x
\end{aligned}
$$

and

$$
\begin{aligned}
& F^{\text {Cellcn }}\left(f_{10}, f_{11}, \phi_{2}\right) \\
& =\lambda_{3} \int\left(\int\left(K_{\sigma_{2}}(x-y)\left|I-f_{11}(x)\right|^{2} H\left(\phi_{1}(x) H\left(\phi_{2}(x)\right) \mathrm{d} y\right) \mathrm{d} x\right)\right.
\end{aligned}
$$


$+\lambda_{4} \int\left(\int\left(K_{\sigma_{2}}(x-y)\left|I-f_{10}(x)\right|^{2} H\left(\phi_{1}(x)\left(1-H\left(\phi_{2}(x)\right)\right) \mathrm{d} y\right) \mathrm{d} x\right)\right.$

$+v_{2} \int\left|\nabla H\left(\varphi_{2}(x)\right)\right| H\left(\varphi_{1}(x)\right) \mathrm{d} x$.

The precondition of eq. (14) is to ensure that the value $\phi_{1}$ after eq. (13) takes positive values in the cell part and negative values in background. This condition can be met by initializing $\phi_{1}$ using simple intensity statistics.

The last terms in functions (13) and (14) are the lengths of the contours. These lengths are often substituted by $\int \delta(\phi) \mid \nabla(\phi) \mathrm{d} x \quad[9,10]$ in which $\delta$ is the Dirac delta function [28]. In practice, the Heaviside function $H$ and its derivative $\delta$ are approximated by

$$
\begin{gathered}
H_{\varepsilon}(x)=\frac{1}{2}\left[1+\frac{2}{\pi} \arctan \left(\frac{x}{\pi}\right)\right], \\
\delta_{\varepsilon}(x)=H_{\varepsilon}^{\prime}(x)=\frac{1}{\pi} \frac{\varepsilon}{\varepsilon^{2}+x^{2}} .
\end{gathered}
$$

To accelerate the calculation process, $\mathrm{Li}$ [15] added a level set regularization term to preserve the regularity of the level set function. An example of the regularization terms that can be used is

$$
P(\phi)=\int \frac{1}{2}(|\nabla \phi(x)|-1)^{2} \mathrm{~d} x .
$$

After these modifications, eqs. (13) and (14) become

$$
\begin{aligned}
& F^{\text {Cellbc }}\left(g_{0}, g_{1}, \phi_{1}\right) \\
& =\lambda_{1} \int\left(\int\left(K_{\sigma_{1}}(x-y)\left|I G-g_{1}(x)\right|^{2} H_{\varepsilon}\left(\phi_{1}(x)\right) \mathrm{d} y\right) \mathrm{d} x\right. \\
& \quad+\lambda_{2} \int\left(\int\left(K_{\sigma_{1}}(x-y)\left|I G-g_{1}(x)\right|^{2}\left(1-H_{\varepsilon}\left(\phi_{1}(x)\right)\right) \mathrm{d} y\right) \mathrm{d} x\right. \\
& \quad+v_{1} \int \mid \nabla H_{\varepsilon}\left(\phi_{1}(x)\right) \mathrm{d} x+\mu_{1} \int \frac{1}{2}\left(\left|\nabla \phi_{1}(x)\right|-1\right)^{2} \mathrm{~d} x
\end{aligned}
$$

and

$$
\begin{aligned}
& F^{\text {Cellcn }}\left(f_{10}, f_{11}, \phi_{2}\right) \\
& =\lambda_{3} \int\left(\int \begin{array}{l}
\left(K_{\sigma_{2}}(x-y)\left|I-f_{11}(x)\right|^{2}\right. \\
H_{\varepsilon}\left(\phi_{1}(x) H_{\varepsilon}\left(\phi_{2}(x)\right) \mathrm{d} y\right) \mathrm{d} x
\end{array}\right) \\
& +\lambda_{4} \int\left(\int \begin{array}{l}
\left(K_{\sigma_{2}}(x-y)\left|I-f_{10}(x)\right|^{2} H_{\varepsilon}\left(\phi_{1}(x)\right)\right. \\
\left.\left(1-H_{\varepsilon}\left(\phi_{2}(x)\right)\right) \mathrm{d} y\right) \mathrm{d} x
\end{array}\right) \\
& +v_{2} \int\left|\nabla H_{\varepsilon}\left(\varphi_{2}(x)\right)\right| H_{\varepsilon}\left(\varphi_{1}(x)\right) \mathrm{d} x \\
& +\mu_{2} \int \frac{1}{2}\left(\left|\nabla \varphi_{2}(x)\right|-1\right)^{2} H_{\varepsilon}\left(\varphi_{1}(x)\right) \mathrm{d} x,
\end{aligned}
$$

in which $\mu$ is a positive coefficient.

\subsection{Energy minimization}

We use the standard gradient descent method to minimize the energy function in eq. (15) and use $\frac{\partial F}{\partial \phi}$ to represent the Gateaux derivative of the function $F$ :

$$
\frac{\partial \phi}{\partial t}=-\frac{\partial F}{\partial \phi}
$$

which represents the gradient flow that minimizes the function $F$. According to [29], let

$$
g_{i}(x)=\frac{K_{\sigma}(x) *\left[M_{i}^{\varepsilon}\left(\phi_{1}(x)\right) I G(x)\right]}{K_{\sigma}(x) * M_{i}^{\varepsilon}\left(\phi_{1}(x)\right)}, i=1,2,
$$

in which $M_{1}(\phi)=H(\phi), M_{2}(\phi)=1-H(\phi)$.

Keeping $g_{1}$ and $g_{2}$ fixed, we can obtain

$$
\begin{aligned}
\frac{\partial \phi_{1}}{\partial t}= & -\delta_{\varepsilon}\left(\phi_{1}\right)\left(\lambda_{1} e_{1}-\lambda_{2} e_{2}\right) \\
& +v_{1} \delta_{\varepsilon}\left(\phi_{1}\right) \operatorname{div}\left(\frac{\nabla \phi_{1}}{\left|\nabla \phi_{1}\right|}\right)+\mu_{1}\left(\nabla^{2} \phi_{1}-\operatorname{div}\left(\frac{\nabla \phi_{1}}{\left|\nabla \phi_{1}\right|}\right) .\right.
\end{aligned}
$$

From eq. (18), we can obtain

$$
e_{i}(x)=\int K_{\sigma}(y-x)\left|I G(x)-g_{i}(y)\right|^{2} \mathrm{~d} y, i=1,2 .
$$

Because the minimization of eq. (19) occurs after the computation of eq. (22), $\phi_{1}$ and $H\left(\phi_{1}\right)$ are known. The calculation is identical to that used in evaluating eq. (18):

$$
\begin{aligned}
\frac{\partial \phi_{2}}{\partial t}= & -\delta_{\varepsilon}\left(\phi_{2}\right) H_{\varepsilon}\left(\phi_{1}\right)\left(\lambda_{3} d_{1}-\lambda_{4} d_{2}\right) \\
& +v \delta_{\varepsilon}\left(\phi_{2}\right) \operatorname{div}\left(\frac{\nabla \phi_{2}}{\left|\nabla \phi_{2}\right|}\right)+\mu\left(\nabla^{2} \phi_{2}-\operatorname{div}\left(\frac{\nabla \phi_{2}}{\left|\nabla \phi_{2}\right|}\right),\right.
\end{aligned}
$$

in which $d_{i}(x)=\int K_{\sigma}(y-x)\left|I(x)-f_{i}(y)\right|^{2} \mathrm{~d} y, i=1,2$, and $f_{i}(x)=\frac{K_{\sigma}(x) *\left[M_{i}^{\varepsilon}\left(\phi_{2}(x)\right) I(x)\right]}{K_{\sigma}(x) * M_{i}^{\varepsilon}\left(\phi_{2}(x)\right)}, i=1,2$.

After calculating $\phi_{1}$, to speed up the computation we initialized $\phi_{2}$ with positive values in the potential nucleolus regions and with negative values in the potential cytoplast regions.

\section{Implementation and analysis}

\subsection{Implementation}

In this model, we first combined the information of the original image $I$ and its gradient $G$ with a proper ratio to produce a new image $I G$. $I G$ contains the information about the image gradients. Based on the cell image feature specified in eq. (3), the gradient of a cell image is larger than that of background. Therefore, we can differentiate cytoplast and background in the new image more easily than when using only the original image. Using the gradient informa- 
tion can be especially advantageous when the intensity difference between cytoplast and background is small.

The first step is to initialize the level set function $\phi_{1}$. In this work, a statistical method was used to predict the potential cell region and background region, and set the initial value of $\phi_{1}$ to be positive in the potential cell region and negative in the potential background region. Afterwards, the contour $C_{1}$ was obtained by iteratively solving eq. (22).

Next, using the statistical method, we initialize the second level set function $\phi_{2}$ to image $I$. We restricted the initialization to the area inside $C_{1}$. Within this area, the value of the potential nucleolus region is set to be positive and that of the potential cytoplast region to be negative. After this initialization, the second contour $C_{2}$ that separates cytoplast and nucleolus can be obtained through iteration of eq. (24).

The two iterations are nearly identical. When multiple cell images are present, pipeline processing can be used to enhance the efficiency because the second iteration is based on the first iteration.

\subsection{Experimental results}

The proposed method was tested on real cell images that were acquired in our laboratory. Unless otherwise specified, in this paper we used the following parameters for processing the images: $\sigma=3.0, \lambda_{1}=\lambda_{2}=1.0$, time step $\Delta t=0.1, \mu=1$. As described in [19], a smaller scale $\sigma$ produces more accurate localization of object boundaries. In contrast, a larger $\sigma$ value can produce results that are more independent of the location of the initial contours. All experiments were performed on a workstation with Intel ${ }^{\circledR}$ Core $^{\mathrm{TM}} 2$ Duo CPU E8200@2.66 GHz, 2.67 GHz, 3.40 GHz CPU and 3.25 GB memory. The workstation operated under Microsoft Win- dows XP Service Pack 2. Matlab 7.6.0 was used for processing.

Figure 2 shows an example of the different stages of the segmentation process that we used. Figure 2(a) is the original image; Figure 2(b) is the gradient of the original image (a). As described previously, the gradient within the cell is much bigger than that of the background; Figure 2(c) shows a composite image which amplifies the difference between the cell and background; Figure 2(d) is the initial level set value of the first function; Figure 2(e) is the result of the iteration of the first level set function; Figure 2(f) is the final result for the first level set function after multiple iterations; Figure $2(\mathrm{~g})$ is the initial level set value of the second function; Figure 2(h) is the result of the iteration of the second level set function; Figure 2(i) is the final image of the second level set function after all iterations. From Figure 2(i), it can be appreciated that the nucleolus is correctly segmented. The image size for Figure 1 was $116 \times 160$ pixels. The total processing time to obtain the final segmentation result was approximately $1.125 \mathrm{~s}$.

Figure 3 is an example of the segmentation process for a cell image that is more complicated than the image in Figure 2. As in Figure 2, (a) is the original image and (b) is the gradient of the original image. Again, the gradient within the cell is noted to be much bigger than that of the background; (c) is a combination of (a) and (b) and further amplifies the difference between cell and background; (d) is the initial level set value of the first function; (e) is the result of the iteration of the first level set function; (f) is the final result of the first level set function; (g) is the initial level set value of the second function; (h) is the result of the iteration of the second level set function; (i) is the final result of the second level set function. As for the images in Figure 2, the nucleolus is correctly segmented. The size of
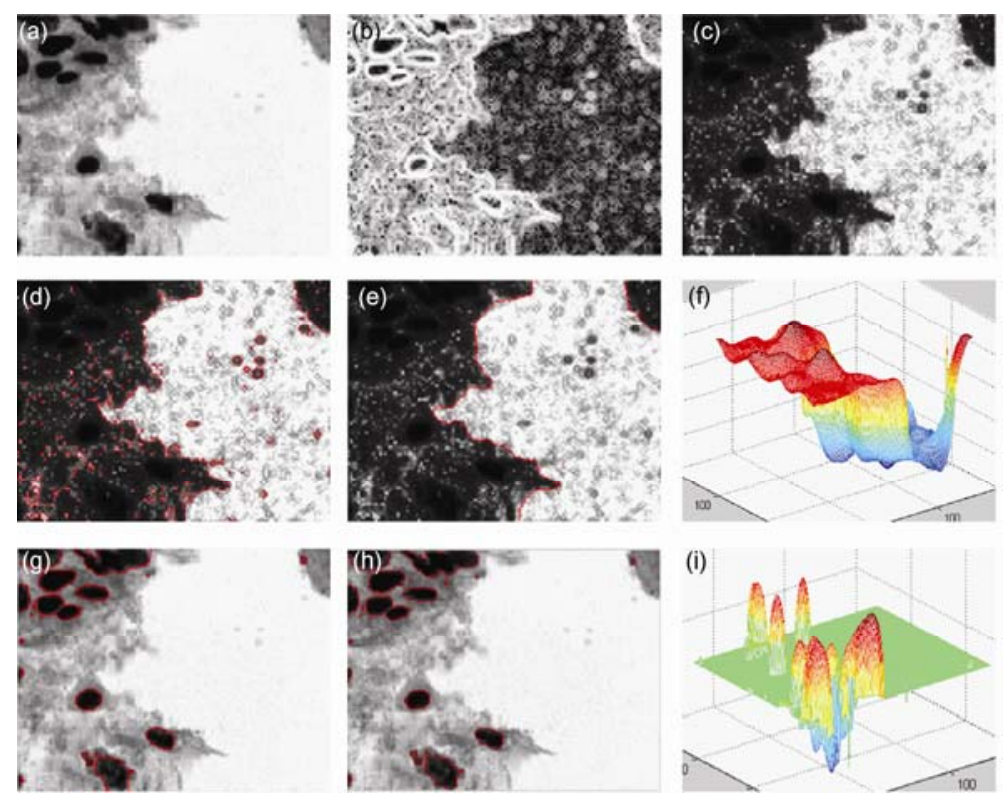

Figure 2 (a) Cell image; (b) gradient; (c) combination of (a) and (b); (d), (e) the 1st segmentation; (g)-(i) the 2nd segmentation. 
the images in Figure 3 was $104 \times 129$ pixels. The total processing time was approximately 1.318 .

\subsection{Comparison with other methods}

Our proposed method has several potential advantages when compared with other previously published methods. First, the model and algorithm we used are implemented under the level set framework. Second, the different features of cells are used to generate an energy conduction model. As a result, our method can produce finer segmentation results even when the intensity difference between cytoplast and background is small. Figure 4(a) shows an example whereby a conventional level set method failed to produce a correct segmentation of cytoplasm and background because of an insufficient intensity difference between the two regions. In contrast, our method was able to overcome this limitation and properly segment the two regions (Figure 4(b), (c)). Finally, our method uses statistical information to initialize the level set function. As a result, the two iterations can be pipelined in execution, allowing a much shorter processing time. In cases where multiple images need to be segmented, the first and second iterations can even performed on different computers.
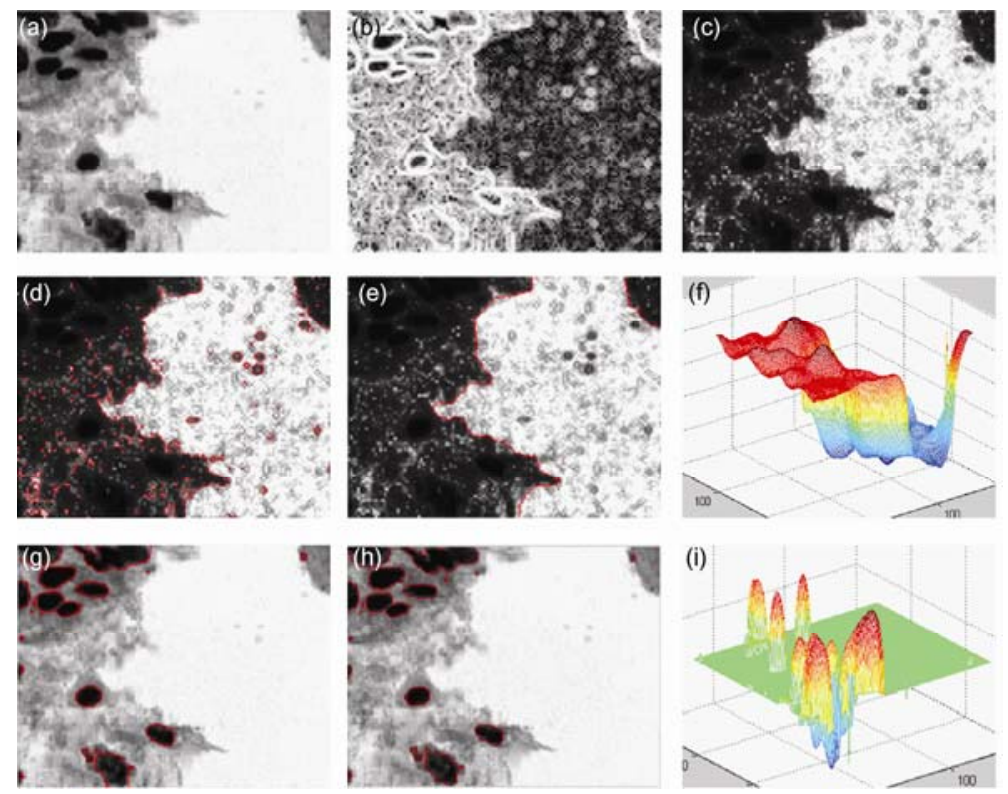

Figure 3 (a) Cell image; (b) gradient image; (c) combination of (a) and (b); (d), (e) the 1st segmentation; (g), (i) the 2nd segmentation.
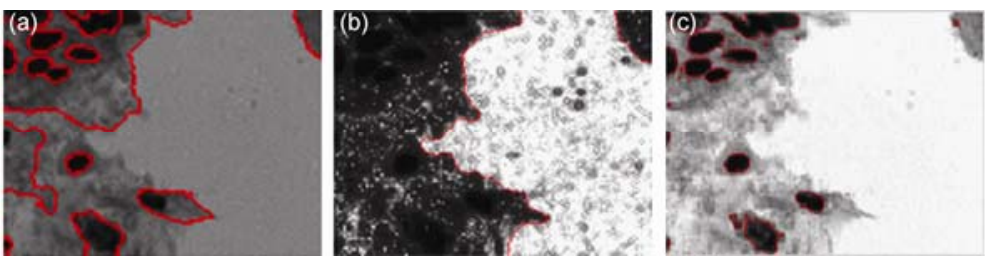

Figure 4 (a) Result segmented with the conventional level set method; (b), (c) result using the proposed new method.

\section{Conclusions}

In summary, we analyzed the different features of a cell image and proposed an energy conduction model to improve cell image segmentation. In real cell images that can be challenging for conventional level set based methods, our proposed method was shown to be capable of detecting the small intensity difference between the different cell regions and producing correct image segmentation results. The proposed algorithm was found to be efficient and can be easily implemented on multiple computers for further improvement in the processing speed.

This work was supported by the National Basic Research Program of China (2011CB707701), the National Natural Science Foundation of China (60873124), the Joint Research Foundation of Beijing Education Committee (JD100010607), the International Science and Technology Supporting Plan (2008BAH26B00) and the Zhejiang Service Robot Key Lab (2008E10004).

1 Semmlow J. Biosignal and Biomedical Image Processing. New York: Marcel Derkker, Inc, 2004

2 Yao L, Liu J, Xie Y, et al. Medical image segmentation based on cel- 
lular neural network. Sci China Ser F-Inf Sci, 2001, 44: 68-72

3 Ma Y, Dai R, Li L, et al. Image segmentation of embryonic plant cell using pulse-coupled neural networks. Chinese Sci Bull, 2002, 47: $167-172$

$4 \mathrm{Li} \mathrm{M}, \mathrm{Su}$ Y, Meng A. Induction of cranial and posterior trunk neural crest by exogenous retinoic acid in zebrafish. Chinese Sci Bull, 2002, 47: 1105-1107

5 Li X, Tian Z. Multiscale stochastic hierarchical image segmentation by spectral clustering. Sci China Ser F-Inf Sci, 2007, 50: 198-211

6 Sheng H, Xiong Z, Weng J, et al. An approach to detecting abnormal vehicle events in complex factors over highway surveillance video. Sci China Ser E-Tech Sci, 2008, 51(suppl ii): 199-208

7 Yang F, Gao Y, Zhang Y, et al. Developing a microfluidic-based system to quantify cell capture efficiency. Sci China Ser C-Life Sci, 2009, 52: 173-181

8 Stanley O, James A S. Fronts propagating with curvature dependent speed: Algorithms based on Hamilton-Jacobi formulations. J Comput Phys, 1988, 79: 12-49

9 Tony C, Luminita V. Active contours without edges. IEEE Trans Image Proc, 2001, 10: 266-276

10 Luminita V, Tony C. A multiphase level set framework for image segmentation using the Mumford and Shah model. Int J Computer Vis, 2002, 50: 271-293

11 Mumford D, Shah J. Optimal approximations by piecewise smooth functions and associated variational problems. Commun Pure Appl Math, 1989, 42: 577-685

12 Chen J, Tian J, Xue J, et al. Level set method with multi-speed-function and its application in segmentation of medical images. J Software, 2007, 18: 842-849

13 Siddhartha B, Ujjwal M, Paramartha D. Multilevel image segmentation with adaptive image context based thresholding. Appl Soft Comput, 2011, 11: 946-962

14 Kaihua Z, Lei Z, Huihui S. Active contours with selective local or global segmentation: A new formulation and level set method. Image
Vision Comput, 2010, 28: 668-676

15 Lei H, Zhigang P, Bryan E. A comparative study of deformable contour methods on medical image segmentation. Image Vision Comput, 2008, 26: 141-163

16 Duan C, Ma J. Medical image segmentation based on level set method. J Software, 2009, 20: 1106-1116

17 Wu K, Hao L, Wang C, et al. Level set interface treatment and its application in Euler method. Sci China Ser G, 2009, 39: 1204-1213

18 Wan C, Yuan Z, Miao Z. A static camera moving object segmentation algorithm based on active contour and Gauss background model. Sci China Ser F, 2009, 39: 391-396

19 Li C. Minimization of region-scalable fitting energy for image segmentation. IEEE Trans Image Proc, 2008, 17: 1940-1949

20 Caselles V. Geodesic active contours. Int J Comput Vis, 1997, 22: 61-79

21 Kass M. Snakes: Active contour models. Int J Comput Vis, 1987, 1: 321-331

22 Kichenassamy S. Gradient flows and geometric active contour models. Proc 5th Int Conf Comput Vis, 1995: 810-815

23 Kimmel R. Finding shortest paths on surfaces using level set propagation. IEEE Trans Pattern Anal Match Intell, 1995, 17: 635-640

24 Vasilevskiy A. Flux-maximizing geometric flows. IEEE Trans Pattern Anal Mach Intell, 2002, 24: 1565-1578

25 Paragios N. Geodesic active regions and level set methods for supervised texture segmentation. Int J Comput Vis, 2002, 46: 223-247

26 Ronfard R. Region-based strategies for active contour models. Int J Comput Vis, 1994, 13: 229-251

27 Samson C. A variational model for image classification and restoration. IEEE Trans Patt Anal Mach Intell, 2000, 22: 460-472

28 Li C. Level set evolution without re-initialization: A new variational formulation. Proc IEEE Conf Comput Vis Pattern Recogn, 2005, 1: $430-436$

29 Li C. Implicit active contours driven by local binary fitting energy. CVPR, 2007: 1-7

Open Access This article is distributed under the terms of the Creative Commons Attribution License which permits any use, distribution, and reproduction in any medium, provided the original author(s) and source are credited. 